ARTICULOS ORIGINALES

\title{
Estudio preliminar de los flebótomos de Villeta y Quebradanegra, Cundinamarca: sus implicaciones en salud pública
}

\author{
Raúl H. Pardo ${ }^{1}$, Sandra Farieta ${ }^{2}$, Leonard E. Munstermann ${ }^{3}$, Cristina Ferro ${ }^{1}$
}

\begin{abstract}
Resumen
Con el fin de preseleccionar los posibles vectores de leishmaniasis cutánea en Villeta y su área rural más cercana, se hizo un reconocimiento de las especies del género Lutzomyia. Los insectos se capturaron durante la noche con cebo humano, cebo animal, trampas Shannon y trampas CDC y, durante el día, por aspiración directa en los sitios de reposo. Se encontraron ocho especies en los 497 insectos recolectados; las más abundantes fueron $\mathrm{Lu}$. ovallesi $(48,7 \%)$, Lu. gomezi $(38,5 \%)$ y $\mathrm{Lu}$. lichyi $(8 \%)$, especies reconocidas como antropofílicas y con antecedentes de ser vectoras o posibles vectoras. Lu. gomezi fue la especie más abundante en las capturas con cebo humano, con una fuerte tendencia peridomiciliaria por lo que podría estar implicada en la transmisión de leishmaniasis en toda el área de estudio. Lu. ovallesi fue más abundante en los bosques poco intervenidos del área rural, por lo que es posible que juegue un papel como vector más importante en esta área. $L u$. lichyi se presentó, principalmente, en el bosque aunque llegó hasta el peridomicilio. Finalmente, es necesario iniciar estudios detallados para aclarar el papel que, como vectores, puedan tener las especies de Lutzomyia antes mencionadas en Villeta y el área rural adyacente.
\end{abstract}

\section{Summary}

To predetermine the possible vectors of cutaneous leishmaniasis, a pilot-study was carried out in the urban and peripheral areas of Villeta, a small town in the central Colombian coffee-growing region, to investigate the composition and distribution of sandflies of the genus Lutzomyia. Sandflies were sampled at night using Shannon trap, CDC light trap and mouth aspirator on human and animal bait. Day-time resting sites were sampled by mouth aspirator. Eight species were found amongst the 497 sandflies collected; Lu. ovallesi $(48.7 \%)$, Lu. gomezi $(38.5 \%)$ and Lu. lichyi $(8 \%)$, being the most abundant, are known to be anthrophophilic species and have already been reported as being vectors or suspected vectors of cutaneous leishmaniasis. Lu. gomezi was the most abundant in collections using human bait, having high preference for peridomestic habitats. Thus, this species may be implicated in disease transmission in the studied area. Lu. ovallesi was the most abundant in the less disturbed forests of the adjacent rural areas and so, this species may be the main vector in this area. Lu. lichyi was present mainly in forests, although a few individuals were found in the peridomiciliary samples. Finally, it is necessary to initiate studies to define the role that these vectors may have within Villeta and the surrounding rural zones.

1 Laboratorio de Entomología, Instituto Nacional de Salud, Santa Fe de Bogotá, Colombia.

2 Secretaría de Salud de Cundinamarca, Santa Fe de Bogotá, Colombia.

3 Department of Epidemiology and Public Health, Yale University School of Medicine, New Haven, Conn., U.S.A. 
El presente trabajo se desarrolló por solicitud del Ministerio de Salud y de la Secretaría de Salud de Cundinamarca a raíz del aumento en el número de casos de leishmaniasis cutánea (LC) en el municipio de Villeta y en otros cercanos a éste como Quebradanegra, Nocaima y Sasaima, además de la presencia de casos urbanos de esta enfermedad. Mientras que, en 1995, se presentaron 22 casos de LC en estos municipios, con excepción de Sasaima, en el primer semestre de este año se registraron 36 casos de los cuales 4 fueron casos urbanos de Villeta $(1,2)$.

La LC es una enfermedad causada por protozoarios del género Leishmania (Kinetoplastida: Trypanosomatidae) que se transmite al hombre únicamente por la picadura de las hembras de algunas especies de flebótomos (Diptera: Psychodidae) que, en el Nuevo Mundo pertenecen al género Lutzomyia. Los flebótomos se infectan cuando toman sangre de un reservorio hospedero, que incluye al hombre y los animales domésticos y salvajes (3).

En Latinoamérica, la epidemiología de la enfermedad es compleja por lo que se presentan dificultades en el desarrollo de las investigaciones. Como inconvenientes principales, se tienen entre otros: a) la existencia de varias especies de Leismania que afectan al hombre, las cuales se presentan en forma simpátrica en algunas regiones; b) la dificultad en la identificación de los flebotómos, ya que se han descrito cerca de 400 especies (4), de las cuales aproximadamente, $20 \%$ son antropofílicas y, de éstas, la mitad $(10 \%)$ se consideran vectoras de enfermedades (5); c) los bajos porcentajes de infeccción con Leishmania en los vectores y la dificultad en el aislamiento de algunas especie de Leishmania a partir del insecto vector; d) el poco conocimiento sobre la ecología de los vectores y de los reservorios.

En Colombia, la LC es endémica; ha sido informada en casi todas las regiones del país, desde 0 hasta los $1.700 \mathrm{msnm}$ (6). Hasta el momento, se han aislado de pacientes, seis especies de Leishmania causantes de LC: $L$. braziliensis, $L$. panamensis, $L$. guyanensis, $L$. amazonensis, L. mexicana y L. colombiensis; el mayor número de aislamientos se ha hecho de dos primeras $(6,7)$; éstas se presentan, generalmente, en bosques primarios, secundarios y en cafetales. En cuanto a los flebótomos, se han registrado 128 especies del género Lutzomyia de las cuales cerca de la mitad tienen informes de ser antropofílicas, dentro o fuera del país; y de éstas, sólo se ha logrado el aislamiento e identificación del parásito en cuatro especies: $L$. braziliensis de Lu. spinicrassa, L. panamensis de Lu. trapidoi, $L$. colombiensis de $L u$. hartmanni y $L$. guyanensis de Lu. umbratilis (6-8).

Para Cundinamarca, se han aislado dos especies de Leishmania de pacientes: $L$. braziliensis y L. panamensis (6). Aunque se han hecho algunas recolecciones puntuales de flebótomos en el departamento (9), en el área de Villeta sólo está informada de Lu. ovallesi (10). Esta especie ha sido incriminada como vectora de L. braziliensis y L. guyanensis en otros países $(11,12)$.

Los objetivos del presente trabajo fueron el hacer un reconocimiento preliminar de las especies de Lutzomyia de Villeta y de la zona rural más cercana, para seleccionar posibles vectores de leishmaniasis cutánea en el área teniendo en cuenta su abundancia, distribución y antropofilia de las especies encontradas. Adicionalmente, se buscaron insectos infectados con parásitos flagelados.

\section{Materiales y métodos}

El trabajo se desarrolló en barrios de la periferia de Villeta y en el área rural del municipio de Quebradanegra con el que limita por el norte, entre el 24 y el 28 de junio de 1996. Villeta está ubicada sobre terreno montañoso, en el lado occidental de la cordillera oriental, a $799 \mathrm{msnm}$, con una temperatura promedio de $24^{\circ} \mathrm{C}$ y un promedio de precipitación anual entre 1.500 y $2.000 \mathrm{~mm}$. Pertenece a la zona de vida del bosque húmedo premontano (bh PM) (13); hace parte de la zona cafetera. Como actividades económicas sobresalen la agricultura (caña panelera, café, maíz, yuca, plátano y frutales), la ganadería y el turismo. 
Sitios de muestreo: se seleccionaron ocho sitios, teniendo en cuenta las viviendas humanas, los bosques cercanos a éstas y su localización en el área rural o urbana. Se trabajaron tres ambientes generales: intradomicilio, peridomicilio y bosque. Cuatro sitios de muestreo se ubicaron en el área rural de la vereda La Concepción del municipio de Quebradanegra, a $20 \mathrm{~km}$ aproximadamente del casco urbano de Villeta.

El primer sitio correspondió a la casa ubicada en la finca El Vergel (casa 1); el segundo, a la casa de la finca Buenavista (casa 2); el tercero fue un bosque secundario cercano a las dos casas (bosque 1-2), a $100 \mathrm{~m}$ de la casa 1 y a $40 \mathrm{~m}$ de la casa 2; era un cafetal descuidado con algunos árboles como sombrío, de aproximadamente $20 \mathrm{~m}$ de altura; el cuarto sitio fue otro bosque secundario (bosque 0 ) más alejado de las casas, aproximadamente a $200 \mathrm{~m}$ de cada una, pero en mejor estado, con indicios de recuperación, relativamente denso y con árboles de una altura aproximada de $20 \mathrm{~m}$.

Los otros cuatro sitios se ubicaron en dos barrios de la periferia de Villeta; los dos primeros pertenecían al barrio Carlos Lleras; estaban formados por la última casa de un extremo del barrio (casa 3), en donde residía un niño de 2 años de edad infectado con LC y por un bosque muy intervenido (bosque 3) que limitaba con la parte posterior de la casa; era una reducida mancha, poco densa, de 15 a $20 \mathrm{~m}$ de altura.

Los últimos dos sitios se escogieron en el barrio Fernando Salazar el cual presentaba características rurales; uno de éstos fue una casa (casa 4) en donde residía una ama de casa de 40 años que también tenía LC y el otro fue un bosque (bosque 4) ubicado a $50 \mathrm{~m}$ de la casa; éste fue el bosque más deteriorado de todos, hacía parte de una pequeña finca de recreo, lo formaba un reducido número de árboles de $10 \mathrm{a}$ $20 \mathrm{~m}$ de altura, dispersos, entre los que se mezclaban algunas plantas de café y ornamentales. El estrato herbáceo estaba reducido a un prado.

Técnicas de muestreo: se utilizaron varios métodos de muestreo aplicados de acuerdo con el tipo de ambiente. Se trabajó con cebo humano en el peridomicilio de las casas y en el bosque; para tal efecto, dos personas con protección adecuada, camisa de manga larga, pantalón largo y con repelente en las partes expuestas, se sentaron a esperar la llegada de los flebótomos durante $30 \mathrm{~min}$ en los sitios escogidos, entre las 18:30 y las 21:30 h.

Los insectos fueron capturados con tubos de aspiración tan pronto como se posaban sobre las personas para evitar que fueran picadas. Se utilizaron trampas Shannon en el peridomicilio y en el bosque con una duración de $30 \mathrm{~min}$ entre las horas antes mencionadas; en cada trampa trabajaron dos personas. Se colocaron trampas CDC en todos los ambientes por $12 \mathrm{~h}$, entre las 18:00 y las 06:00 h, a una altura de $1,5 \mathrm{~m}$ con excepción de los bosques en donde también se colocaron trampas a $10 \mathrm{~m}$ de altura en la parte inferior del dosel.

Dos personas hicieron capturas sobre cebo animal en el peridomicilio: en marranos, en el área rural, y en gallinas, en los barrios periféricos de Villeta, por $30 \mathrm{~min}$ en la noche y por el mismo tiempo durante las primeras horas de la mañana.

Durante el día, dos personas por sitio buscaron en los troncos de los árboles de los bosques por un tiempo de 40 a 60 min. Adicionalmente, entre las $6: 30$ y 8:00 $\mathrm{h}$, una persona revisó por un tiempo de $15 \mathrm{~min}$ las paredes de los intradomicilios.

Los muestreos se realizaron simultáneamente en el área rural y en los barrios de la periferia de Villeta.

Tratamiento de los flebótomos capturados: la mayoría de los flebótomos capturados se preservaron en viales con alcohol al $70 \%$, debidamente rotulados $\mathrm{y}$, posteriormente, se identificaron en el Laboratorio de Entomología del Instituto Nacional de Salud, previo tratamiento de aclaración en $\mathrm{KOH}$ al $20 \%$ y fenol líquido. Los insectos capturados en las trampas Shannon, cebo humano y una parte de los capturados en los troncos de árboles y cebo animal se destinaron a la búsqueda de infección con parásitos, para lo cual los especímenes 
capturados vivos se mataron en una solución con detergente suave al $2 \%$; luego, se colocaron en viales de criopreservación que contenían solución protectora de DMSO al 10\% en PBS $(\mathrm{pH} 7,4)$ y se conservaron en nitrógeno líquido hasta su disección en el laboratorio en donde se buscaron las formas flageladas del parásito en el tubo digestivo (8).

\section{Resultados}

Composición de especies: se tomaron 125 muestras de las cuales 55 (44\%) fueron positivas para flebótomos. En éstas, se capturaron 497 insectos distribuidos en ocho especies. Lu. ovallesi fue la especie más abundante $(48,7 \%)$ seguida de Lu. gomezi $(38,5 \%)$; con una amplia diferencia les siguieron Lu. lichyi $(8 \%)$ y Lu. shannoni $(2,4 \%)$. Ocasionalmente, se capturaron ejemplares de Lu. dubitans, Lu. trinidadensis, Lu. carpenteri y una especie del subgénero Helcocyrtomyia (cuadro 1).

Presencia en los ambientes muestreados: a pesar de que se encontraron flebótomos en, prácticamente, todos los puntos de muestreo, la abundancia y el número de muestras positivas fueron bajas. Los valores más altos de abundancia se presentaron con Lu. gomezi en
Cuadro 1. Flebótomos capturados en los barrioperiféricos de Villeta y la zona rural cercana del municipio de Quebradanegra

\begin{tabular}{lrrrr}
\hline \multicolumn{1}{c}{ Especie } & Hembras & Machos & Total & $\%$ \\
\hline Lutzomyia ovallesi & 91 & 171 & $\mathbf{2 4 2}$ & 48.7 \\
Lutzomyia gomezi & 82 & 109 & $\mathbf{1 9 1}$ & 38.5 \\
Lutzomyia lichyi & 12 & 28 & $\mathbf{4 0}$ & 8.0 \\
Lutzomyia shannoni & 2 & 10 & $\mathbf{1 2}$ & 2.4 \\
Lutzomyia dubitans & 2 & 3 & $\mathbf{5}$ & 1.0 \\
Lutzomyia trinidadensis & 0 & 3 & $\mathbf{3}$ & 0.6 \\
Lutzomyia carpenteri & 0 & 1 & $\mathbf{1}$ & 0.2 \\
Helcocytomyia sp. & 3 & 0 & $\mathbf{3}$ & 0.6 \\
Total & $\mathbf{1 9 2}$ & $\mathbf{3 2 5}$ & $\mathbf{4 9 7}$ & 100.0 \\
\hline
\end{tabular}

las marraneras del peridomicilio (19 insectos/ hombre/h) y en trampas CDC a $10 \mathrm{~m}$ de altura en el bosque (11 insectos/trampa/12h) y con $L u$. ovallesi en el bosque, capturadas en troncos de árbol (22 insectos/hombre/h). Estas dos especies se encontraron en todos los ambientes muestreados, siendo las únicas que entraron en el intradomicilio. Lu. lichyi y $L u$. shannoni se presentaron, principalmente, en el bosque aunque también llegaron al peridomicilio. Las demás especies se encontraron básicamente en el bosque (cuadro 2). De otra parte, ningún flebótomo fue capturado en los gallineros ni en las paredes de los intradomicilios.

Cuadro 2. Abundancia de los flebótomos según el ambiente muestreado y la técnica de captura.

\begin{tabular}{|c|c|c|c|c|c|c|c|c|c|}
\hline \multirow[t]{2}{*}{ Sitio } & \multirow[t]{2}{*}{ Técnica de captura } & \multicolumn{7}{|c|}{ \# $\bar{x}$ de Lutzomyia / hombre / hora* } & \multirow[b]{2}{*}{ Helcocyrtomyia sp. } \\
\hline & & ovallesi & gomezi & lichyi & shannoni & dubitans & trinidadensis & carpenteri & \\
\hline \multirow[t]{3}{*}{ Intradomicilio } & Trampa CDC & 1.2 & 1.3 & --- & --- & $\cdots$ & --. & $\cdots$ & $--\cdot$ \\
\hline & Aspiración en paredes & $\cdots$ & $\cdots$ & $\cdots$ & -- & --- & --- & $\cdots$ & $\cdots$ \\
\hline & & & & & & & & & $\cdots$ \\
\hline \multirow[t]{8}{*}{ Peridomicilio } & Cebo humano & $\cdots$ & 2.0 & $\cdots$ & -- & --- & -- & --- & --- \\
\hline & Trampa Shannon & 3.0 & 2.0 & -.- & -- & -- & --- & $-\cdots$ & --- \\
\hline & Trampa CDC & 2.0 & 2.5 & --- & -- & 1.0 & $\cdots$ & $\cdots$ & $\cdots$ \\
\hline & Cebo animal & & & & & & & & --- \\
\hline & Marranos (nocturna) & 1.2 & 19.0 & 1.1 & 0.5 & $\cdots$ & $\cdots$ & $\cdots$ & -- \\
\hline & Marranos (diurna) & 2.0 & 4.0 & -- & --- & --- & -- & --- & -- \\
\hline & Gallinas (nocturna) & --- & -- & --- & $\cdots$ & --- & $\cdots$ & $\cdots$ & -- \\
\hline & Gallinas (diurna) & $\cdots$ & -- & -- & $\cdots$ & $\therefore$ & $\cdots$ & $\cdots$ & --- \\
\hline \multirow[t]{5}{*}{ Bosque } & Cebo humano & 2.0 & 2.7 & --- & 2.0 & --- & $--\cdot$ & $\cdots$ & 6.0 \\
\hline & Trampa Shannon & --- & 2.0 & 2.0 & $-\cdots$ & --- & --- & $\cdots$ & $\cdots$ \\
\hline & Trampa CDC $10 \mathrm{~m}$ & 5.7 & 11.0 & 1.0 & --- & $\cdots$ & $1: 5$ & $--\cdot$ & -- \\
\hline & Trampa CDC $1.5 \mathrm{~m}$ & 4.0 & 2.8 & 1.0 & 1.0 & 1.0 & --- & 1.0 & $\cdots$ \\
\hline & Aspiración tronco de árbol & 22.0 & 2.2 & 4.1 & 1.3 & --- & --- & --- & -- \\
\hline
\end{tabular}

* Para las trampas CDC los valores corresponden a: \# $\bar{x}$ de Lutzomyia/trampa/12 horas. 
Debido a la notoria abundancia de Lu. ovallesiy Lu. gomezi, a continuación se tratarán más detalladamente con base en las capturas con trampas CDC, Shannon y con cebo humano. Considerando que, en la mayoría de los casos, el número de muestras positivas fue mínimo, una o dos, los resultados deben interpretarse con mucho cuidado.

Lu. ovallesi: con base en las capturas con trampas CDC, esta especie parece ser más abundante en los bosques del área rural que en los bosques que limitan con los barrios de la periferia de Villeta; en el bosque 0, fue más abundante a $1,5 \mathrm{~m}$ y en el bosque $1-2$ a $10 \mathrm{~m}$ (figura 1a). Adicionalmente, esta especie se capturó con cebo humano solamente en el bosque 0 . En las casas con trampas CDC, fueron mayores en la casa 1 y en la casa 2 del área rural que en las de los barrios de la periferia de Villeta (figura $1 \mathrm{~b}$ ).

En el área rural, se hicieron capturas tanto en el peridomicilio como en el intradomicilio mientras que en los barrios de la periferia de Villeta solamente fue positivo el intradomicilio de la casa 3. De otra parte, el único sitio positivo con trampa Shannon fue la casa 1. En general, las capturas en las casas fueron mucho menores que las realizadas en los bosques. Al comparar las capturas en las casas con las de sus bosques cercanos, parece que las casas del área rural (casa $i$ y casa 2) cercanas a los bosques con mayor presencia de Lu. ovallesi (bosque 0 y bosque 1-2) tienen también mayor presencia de esta especie (figura la y b).

Lu. gomezi: con base en las capturas con trampas CDC, esta especie fue más abundante en el bosque $1-2$ a $10 \mathrm{~m}$ de altura; en todos los bosques, con excepción del bosque 4 , se presentó de forma similar a $1,5 \mathrm{~m}$ de altura. Sin embargo, con cebo humano, se presentó de forma similar en el bosque $1-2$ y en el bosque 4; también, fue similar su presencia con trampas Shannon en los bosques de los barrios periféricos de Villeta (figura 2a). En cuanto a las capturas en las casas con trampas CDC, éstas fueron similares en las casas del área rural y en la casa 3. En la casa 4, se lograron capturas únicamente con cebo humano y con trampas Shannon (figura 2b). En términos generales, la presencia de Lu. gomezi en los bosques parece ser ligeramente mayor que la encontrada en las casas más cercanas tanto en el área rural como en los barrios de la periferia de Villeta, con la excepción de las capturas con CDC en el bosque 1-2 a $10 \mathrm{~m}$ de altura (figura $2 \mathrm{a} \mathrm{y} \mathrm{b}$ ).

Infección con Leishmania: se disectaron 74 hembras, 33 de Lu. ovallesi, 30 de Lu. gomezi, 7 de Lu. lichyi, 2 de Lu. shannoni y 2 de una especie del subgénero Helcocyrtomyia sin encontrarse infección con flagelados.

\section{Discusión}

A pesar del corto tiempo del estudio y del relativo bajo número de muestras positivas para Lutzomyia, el presente trabajo permite tener una buena aproximación a la composición de especies de la periferia de Villeta y su área rural circundante. Todas estas especies se han informado previamente en Cundinamarca: $L U$. ovallesi, en Villeta, El Colegio, La Mesa y Ricaurte; Lu. gomezi en Ricaurte; Lu. lichyi, en La Mesa; Lu. shannoni, en Puerto Salgar, Tocaima, Guaduas, La Mesa y Caparrapí; Lu. trinidadensis en La Mesa, Puerto Salgar, Tocaima, Caparrapí y Ricaurte; Lu. dubitans y Lu. carpenteri en Ricaurte $(9,10$, datos sin publicar, Laboratorio de Entomología, INS). Cinco de las ocho especies informadas se encuentran, bajo condiciones similares, en el departamento de Norte de Santander: $L u$. gomezi, Lu. ovallesi, Lu. shannoni, Lu. lichyi y Lu. dubitans, aunque con valores de abundancia diferentes; allí las dos primeras especies también son abundantes (14).

Las especies más importantes por su abundancia, presencia en todos los ambientes muestreados, antropofilia y antecedentes como vectores fueron $L u$. gomezi y $L U$. ovallesi quienes representaron $87 \%$ de las especies encontradas. Lu. lichyi y Lu. shannoni, a pesar de no ser abundantes, pueden llegar a tener importancia por su presencia en el peridomicilio y sus antecedentes antropofílicos, especia!mente los de Lu. lichyi. 


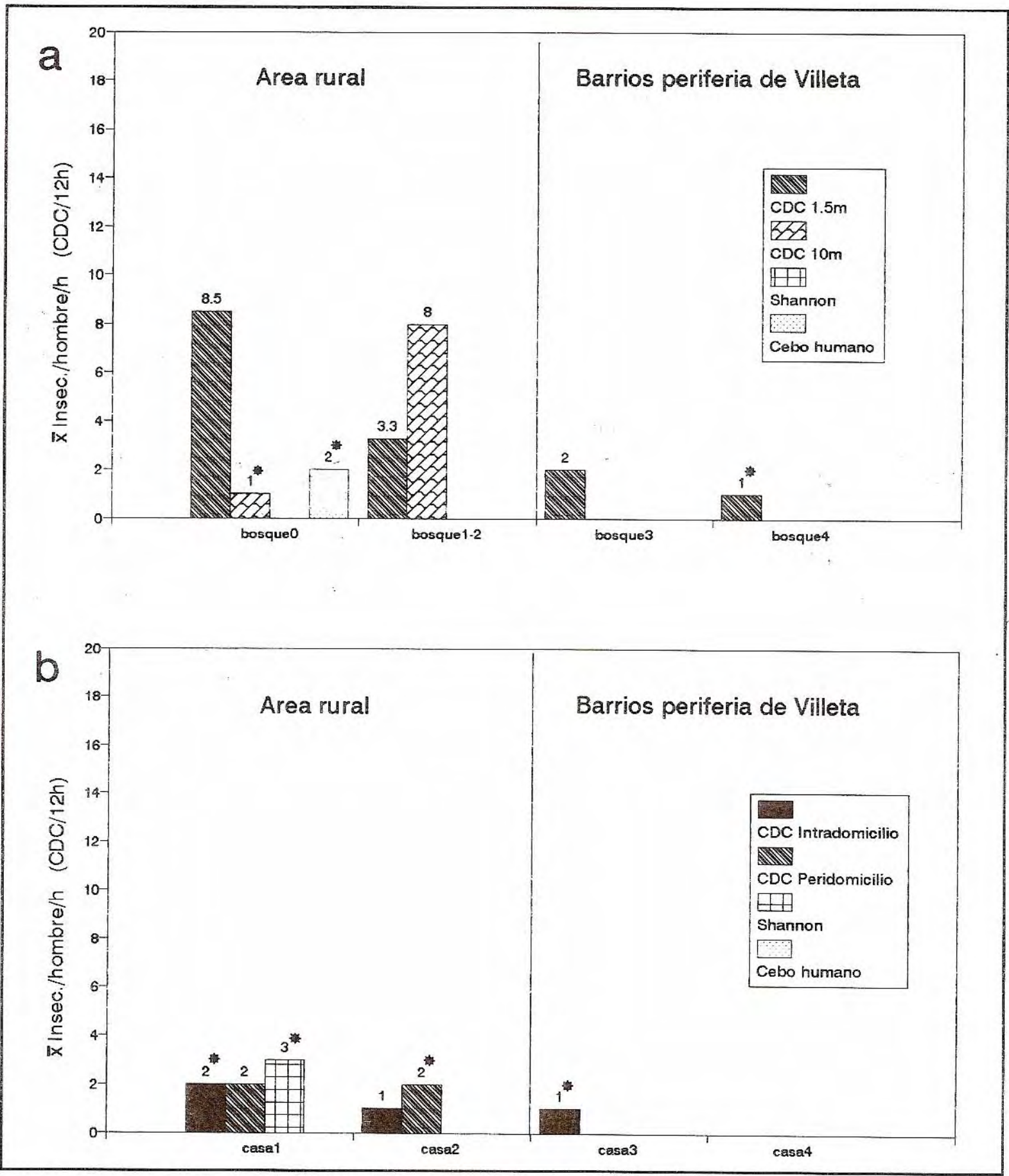

Figura 1. Presencia de Lutzomyia ovallesi en los sitios de muestreo: a) bosques; b) casas. Los bosques más cercanos a cada casa tienen la misma numeración que éstas. $\left({ }^{*}\right)$ El valor corresponde solamente a un dato. 


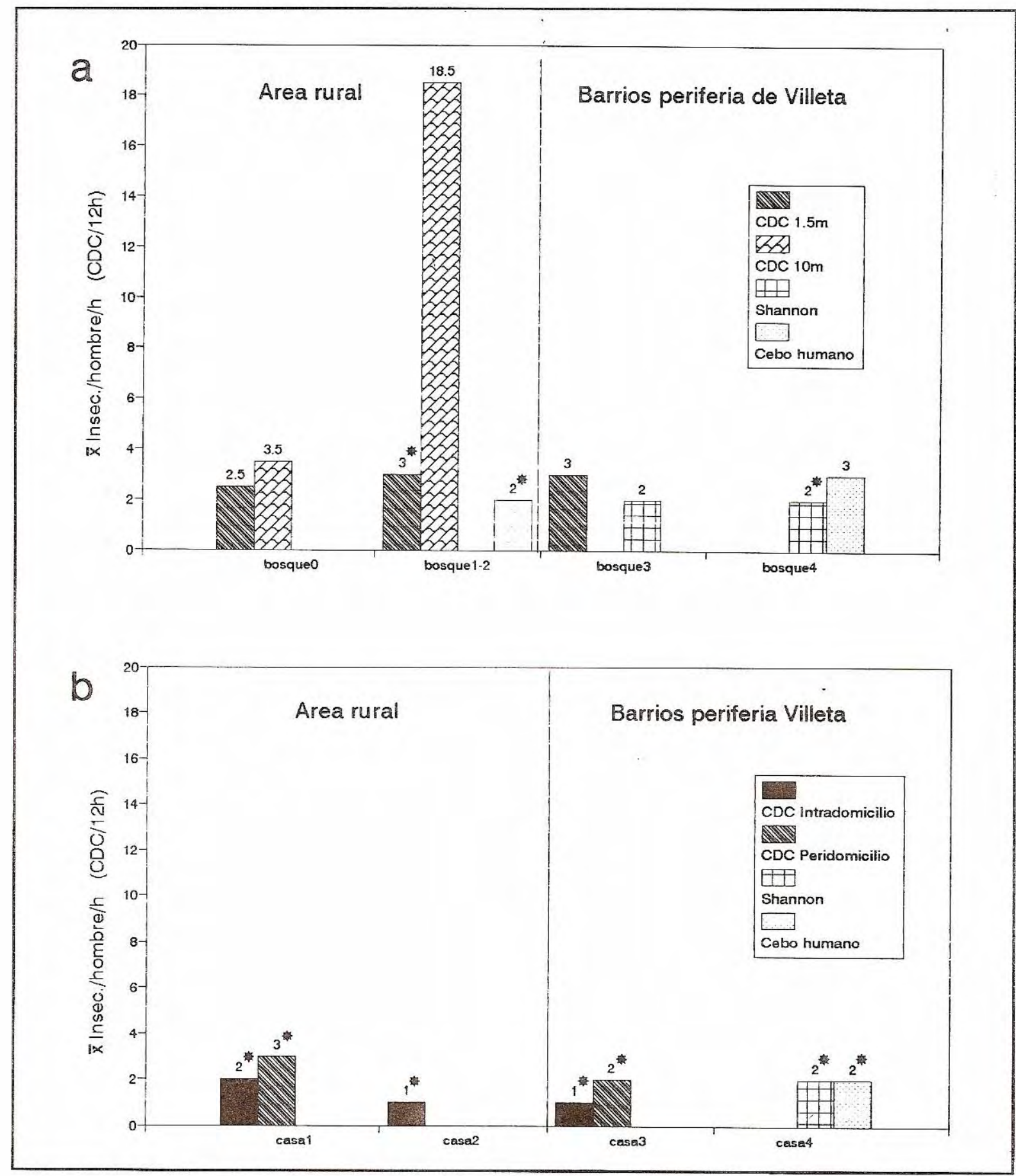

Figura 2. Presencia de Lutzomyia gomezi en los sitios de muestreo: a) bosques; b) casas. Los bosques más cercanos a cada casa tienen la misma numeración que éstas. $\left(^{*}\right)$ El valor corresponde solamente a un dato. 
La preferencia de Lu. ovallesi por los bosques secundarios en relativo buen estado de conservación del área rural (bosque 0 y 1-2) y su presencia en las casas cercanas concuerda con lo observado en Venezuela en donde esta especie se describe como propia del bosque, que no se aproxima a las casas en alta densidad (15). Su hallazgo en alta densidad reposando en troncos de árboles en el bosque, también ha sido informado previamente (14). Su conocida antropofilia se evidenció en su presencia en las capturas con trampas Shannon en el peridomicilio, CDC en el intradomicilio y cebo humano en el bosque.

Lu. gomezi parece ser una especie de mayor adaptación a los ambientes muy intervenidos, pues, su presencia y abundancia no varió drásticamente en bosques poco o muy intervenidos (bosque 0, 1-2 y 3 ) en las casas cercanas (casa 1, 2 y 3), con excepción de las capturas con trampas CDC a $10 \mathrm{~m}$ de altura; esto también concuerda con su notoria abundancia en las marraneras del peridomicilio del área rural. Lo anterior coincide con las observaciones de otros autores que definen a esta especie como de áreas claras y bosques secundarios que están comenzando a recuperarse, con presencia exitosa en el peridomicilio $(15,16)$. En cuanto a la tendencia a presentarse en la parte superior del bosque, ésta ya se ha observado en otros trabajos en Brasil, Panamá y Colombia $(14,17,18)$. De otra parte, su presencia relativamente similar en el bosque más deteriorado (bosque 4) y su casa más proxima (casa 4), lograda únicamente con cebo humano y trampas Shannon, muestra que su tendencia antropofílica es mayor que la de Lu. ovallesi $(14,19)$.

Con relación a $L u$. lichyi y $L u$. shannoni, parecen ser especies del bosque que eventualmente pueden encontrarse en el peridomicilio y presentan cierta tendencia antropofílica. En el Valle del Cauca, Lu. lichyi se ha capturado abundantemente en el intradomicilio (20).

En cuanto a la infección con parásitos, el hecho de no haberse encontrado se explica por el reducido número de hembras disectadas. Esto, teniendo en cuenta que los porcentajes de infección informados hasta ahora en áreas en donde dominan Lu.ovallesi y Lu.gomezi están alrededor de $0,4 \%(21,22)$. Las cuatro especies de Lutzomyia mencionadas en esta discusión han sido relacionadas con la transmisión de leishmaniasis. Lu. ovallesi se ha encontrado infectada con $L$. braziliensis en Guatemala y Venezuela $(11,23)$; en este último país, además, con un híbrido de $L$. brasiliensis $L$. guyanensis (24). Flagelados no identificados se han informado en Colombia y Belice $(8,25)$. $L u$. gomezi se ha encontrado infectada con promastigotas en áreas endémicas de $L$. panamensis en Ecuador y Panamá; además, se ha infectado artificialmente con esta especie de Leishmania $(29,30)$. infección natural con $L$. colombiensis se ha encontrado en Panamá y se han hecho infecciones artificiales (7); este flebótomo también se ha logrado infectar artificialmente con L. braziliensis; se ha encontrado infección con promastigotas no identificadas en ejemplares de Lu. gomezi provenientes de Tolima, Norte de Santander y de la costa pacífica $(8,31,32)$. Lu. shannoni se ha encontrado con flagelados no identificados en Colombia, Guatemala y Panama $(8,21,27,31)$. $L u$. lichyi ha sido infectada artificialmente con $L$. braziliensis (33) y, aunque no se ha encontrado infección natural, es posible que esta especie esté involucrada en la transmisión de leishmaniasis en Colombia $(20,34)$.

Finalmente, con base en los datos obtenidos preliminarmente en el presente estudio y en los antecedentes bibliográficos, puede decirse que en la periferia de Villeta y su zona rural adyacente hay cuatro especies de flebotómos probablemente implicadas en la transmisión de leismaniasis cutánea: Lu. gomezi, Lu. ovallesi, Lu. lichyi y Lu. shannoni. Las dos primeras parecen ser más importantes por su abundancia. Por su tendencia a ocupar áreas intervenidas y mayor antropofilia, Lu. gomezi puede estar implicada tanto en el área rural como en los barrios periféricos de Villeta. En cuanto a $L u$. ovallesi, debido a su presencia más notoria en los bosques secundarios del área rural, es posible que juegue un papel más importante allí, sin descartar que puede tener 
ingerencia sobre los otros ambientes. El papel aparentemente secundario que parecen tener $L u$. lichyi y $L u$. shannoni, por su menor abundancia, puede eventualmente cambiar en otro período del año; cabe anotar que $L u$. shannoni no es una especie muy antropofílica en Colombia. Es probable que $L$. panamensis y $L$. braziliensis sean los parásitos involucrados en la transmisión en el área de estudio. De otra parte, desde el punto de vista de la presencia de probables vectores, es posible que los casos de LC informados en las casas de los barrios periféricos de Villeta se hayan realmente originado allí. Con relación al área rural, es evidente el riesgo que corren sus habitantes, pues, los flebótomos antes mencionados llegan tanto al peridomicilio como al intradomicilio.

Con base en todo lo expuesto, es urgente el desarrollo de un estudio detallado en el que se determine, por lo menos, la especie de parásito, las especies vectoras, su distribución precisa, abundancia estacional y sitios de contacto con el hombre para que, con base en esto, se apliquen las medidas de control adecuadas.

\section{Agradecimientos}

Los autores agradecen la valiosa colaboración en la organización y ejecución del trabajo de campo de la doctora Luz Inés Villarreal del Ministerio de Salud; el apoyo del señor Marco Fidel Suárez y de los técnicos de saneamiento de la Secretaría de Salud de Cundinamarca. Además, agradecemos la colaboración que prestó el Hospital Salazar de Villeta.

Este trabajo se realizó con fondos del proyecto 3100302 del Programa de Investigaciones Biomédicas del Instituto Nacional de Salud en convenio con la Universidad de Yale.

\section{Referencias}

1. Secretaría de Salud de Cundinamarca. Informe anual sobre leishmaniasis; 1995.

2. Secretaría de Salud de Cundinamarca. Estadísticas sobre leishmaniosis, casos informados por el Hospital Salazar, Villeta, hasta junio de 1996.

3. Desjeux P. Human leishmaniases: epidemiology and public health aspects. World Health Statist Quart 1992;45:267-75.
4. Young DG, Duncan M. Guide to the identification and geographic distribution of Lutzomyia sandflies in Mexico, the West Indies, Central and South America (Díptera: Psychodidae). Mem Amer Entomol Inst 1994; (54):1-881.

5. Organización Panamericana de la Salud. Epidemiología, diagnóstico, tratamiento y control de las leishmaniasis en América Latina. OPS/HPC/HCT/94.36; 1994.

6. Corredor A, Kreutzer RD, Tesh RB, Boshell J, Paláu MT, Cáceres E, et al. Distribution and etiology of leishmaniasis in Colombia. Amer J Trop Med Hyg 1990;42(3):206-14.

7. Kreutzer RD, Corredor A, Grimaldi JG, Grot M, Rowton ED, Young DG, et al. Characterization of Leishmania colombiensis sp. $\mathrm{n}$. (Kinetoplastida: Trypanosomatidae), a new parasite infecting humans, animals, and phebotomine sandflies in Colombia and Panamá. Am J Trop Med Hyg 1991;44(6):662-75.

8. Young DG, Morales A, Kreutzer RD, Alexander JB, Tesh RB, Ferro C, Rodríguez C. Isolations of Leishmania braziliensis (Kinetoplastida: Trypanosomatidae) from cryopreserved Colombian sandflies (Díptera: Psychodidae). J Med Entomol 1987;24(5): 587-9.

9. Ferro C, Morrison A, Torres M, Pardo R, Wilson ML, Tesh RB. Species composition and relative abundance of sandflies of the genus Lutzomyia (Diptera: Psychodidae) at an endemic focus of visceral leishmaniasis in Colombia. J Med Entomol 1995;32(4): 527-37.

10. Osorno E, Morales A, Osorno de F, Ferro C. Phlebotominae de Colombia (Diptera: Psychodidae). IX. Distribución geográfica de especies de Brumptomyia franca y Parrot, 1921, y Lutzomyia franca, 1924 encontradas en Colombia S. A. Rev Acad Col Cien Exac Fis Nat 1972; XIV(53):45-68.

11. Rowton ED, De Mata M, Rizzo N, Porter $\mathrm{CH}$, Navis TR. Isolation of Leishmania braziliensis from Lutzomyia ovallesi (Diptera: Psychodidae) in Guatemala. Am J Trop Med Hyg 1992;46(4):465-8.

12. Grimaldi GJR, Tesh RB. Leishmaniases of the New World: current concepts and implications for future research. Clin Microb Rev 1993;230-50.

13. Espinal ST. Zonas de vida o formaciones vegetales de Colombia. Memoria explicativa sobre el mapa ecológico. Bogotá: IGAC;1978.

14. Alexander B, Ferro C, Young D, Morales A, Tesh RB. Ecology of flebotomine sandflies (Diptera: Psychodidae) in a focus of Leishmania (Viannia) braziliensis in northeastern Colombia. Mem Inst Oswaldo Cruz 1992;87(3):387-95.

15. Feliciangeli MD. Ecology of sandflies (Diptera: Psychodidae) in a restricted focus of cutaneous 
leishmaniasis in northern Venezuela. II. Species composition in relation to habitat, catching method and hour of catching. Mem Inst Oswaldo Cruz 1987;82(1):125-31.

16. Porter C, Defoliart GR. The man-biting activity of phlebotomine sandflies (Diptera: Psychodidae) in a tropical wet forest environment in Colombia. Arq Zool S Paulo 1981;30(2):81-158.

17. Azevedo ACR, Bessa LSL, Vilela ML, Rangel EF. Studies on the sandfly fauna of Samuel ecological station, Porto Vehlo municipality, Rondonia state, Brazil. Mem Ins Oswaldo Cruz 1993;88(4):509-12.

18. Chaniotis BN, Correa MA, Tesh BR, Johnson KM. Daily and seasonal man-biting activity of phebotomine sandflies in Panamá. J Med Entomol 1971;8(4):415-20.

19. Christensen HA, Herrer A. Attractivenes of sentinel animals to vectors of leishmaniosis in Panamá. Amer $\mathrm{J}$ Trop Med Hyg 1973;22(5):578-85.

20. Alexander B, Usma MC, Cadena H, Quezada BL, Solart Y, Roa W, et al. Phlebotomine sandflies associated with a focus os cutaneous leishmaniasis in Valle del Cauca, Colombia. Med Vet Entomol 1995;9:273-8.

21. Rowton ED, De Mata M, Rizzo N, Navin TR, Porter $\mathrm{CH}$. Vectors of Leishmania braziliensis in the Peten, Guatemala. Parasitologia 1991;33(suppl.1):501-4.

22. Añez N, Nieves E, Casorla D, Oviedo M, Lugo de Yarbuh A, Valera M. Epidemiology of cutaneous leishmaniasis, in Mérida, Venezuela. Altitudinal distribution, age structure, natural infection and feeding behaviour of sandflies and their relation to the risk of transmission. Ann Trop Med Parasitol 1994;88(3):279-87.

23. Feliciangeli MD. Vectors of leishmanioses in Venezuela. Parasitología 1991;33(Suppl.1):229-36.

24. Bonfante-Garrido R, Spinetti H, Cupillo E, Momen H, Grimaldi G. Lutzomyia ovallesi (Díptera: Psychodidae) as a vector of cutaneous leishmaniasis in Venezuela. Parasitología 1991;33(Suppl.1):99-104.

25. Williams $\mathbf{P}$. Phebotomine sandflies and leishmanioses in British Honduras (Belize). Trans Roy Soc Trop Med Hyg 1970;64(3):317-68.
26. Gómez EAL, Hashiguchi Y. Vectors entomology. 1. Natural infections of sandflies with Leishmania promastigotes. En: Hachiguchi Y, ed. Studies on New World leishmanioses and its transmission, with particular reference to Ecuador. Kochi: Kyowa Printing; 1987; series No.1:70-8.

27. Johnson PT, McConnell E, Hertig M. Natural infections of leptomonad flagellates in Panamanian phlebotomus sandflies. Exp Parasitol 1963;14:107-22.

28. Christensen HA, Fairchild GB, Herrer A, Johnson CM, Young DG, Vásquez de AM. The ecology of cutaneous leishmaniasis in the republic of Panamá. J Med Entomol 1983;20(5):463-84.

29. Walters LL, Chaplin GL, Modi GB, Tesh RB. Ultrastructural biology of Leishmania (Viannia) panamensis (Leishmania braziliensis panamensis) in Lutzomyia gomezi (Díptera: Psychodidae): a natural host-parasite association. Am J Trop Med Hyg 1989; 40(1):19-39.

30. Jaramillo C, Travi B, Montoya J. Vector competence of some neotropical sandflies for the Leishmania (Viannia) braziliensis complex. Med Vet Entomol 1994;8:1-7.

31. Morales A, Corredor A, Cáceres E, Ibagos AL, Rodríguez de C. Aislamiento de tres cepas de leishmania a partir de Lutzomyia trapidoi en Colombia. Biomédica 1981;1(4):198-207.

32. Travi BL, Montoya J, Solarte Y, Lozano L, Jaramillo C. Leishmaniasis in Colombia. I. Studies on the phlebotomine fauna associated with endemic foci in the Pacific coast region. Am J Trop Med Hyg 1988; 39(3):261-6.

33. Warburg A, Montoya-Lerma J, Jaramillo C, CruzRuiz AL, Ostrovska K. Leishmaniasis vector potencial of Lutzomyia species in Colombian coffee plantations. Med Vet Entomol 1991;5:9-16.

34. Montoya J, Jaramillo C, Palma G, Gómez T, Segura I, Travi BL. Report of an epidemic outbreak of tegumentary leishmaniasis in a coffee growing area of Colombia. Mem Inst Oswaldo Cruz 1990;85(1):119-21. 